\title{
Population Synthesis of Massive Close Binary Evolution
}

\author{
J.J. Eldridge
}

\begin{abstract}
Binary population synthesis is the method by which predictions of varied observables of stellar populations can be made from theoretical models of binary stellar evolution. Binary stars have many more possible evolutionary outcomes compared to single stars and the relative rates of the different pathways, such as the rates of different supernova types, depend on uncertain or poorly constrained physics. In this Chapter we describe population synthesis, outline the major uncertainties and discuss the relevant predictions for core-collapse supernovae. After we overview single star evolution we outline the important physical processes that occur in binaries including Roche-lobe overflow, common-envelope evolution and supernova kicks. We also discuss how a synthetic stellar population incorporating interacting binaries can be constructed and how uncertainties, such as the strength of supernova kicks, affect any predictions. We illustrate the process by comparing predictions for the stellar populations in two young star clusters. We then discuss the important predictions from population synthesis for understanding core-collapse supernovae, their delay-time distribution and their progenitor stars. Finally we discuss how we can predict the rate of mergers of compact remnants and thus predict the initial parameters of gravitational wave sources.
\end{abstract}

\section{Introduction}

Supernovae arise from stars. Therefore to understand these explosive death throes we must understand the evolution of stars. While studying a single supernova and its associated progenitor star can be useful, only a handful of events have suitable observational datasets to understand them. An alternative method to gain understanding of supernovae is to study the population of explosions. To do this the population

J.J. Eldridge

The Department of Physics, The University of Auckland, Private Bag 92019, Auckland, New Zealand, e-mail: j.eldridge@auckland.ac.nz 
of supernovae must be modelled. This requires creating a synthetic stellar population of the stellar progenitors that can be compared to the observed population of supernovae. This is performed by population synthesis.

One of the main problems of population synthesis is how to ensure the accuracy of an individual stellar model and the resultant population. This is especially true when attempting to model supernovae as every phase of evolution must be calculated and thus many different uncertainties can limit the confidence of any predictions. One direct method is to compare models to the Sun or other well observed stars. For the latter case the best examples are double-lined spectroscopic-eclipsing binary stars, because for these stars it is possible to accurately deduce their mass, radius and surface temperature of the stars [1, 2]. However these primarily only provide tests of stellar structure, with many containing only main-sequence stars, and are thus limited in what they can tell us about the broader picture of stellar evolution.

To achieve a broader view the next logical step is to study populations of stars, such as those in a star cluster with similar ages and initial compositions. The different evolutionary states of stars in a population is due to their initial mass. Population synthesis is the method of combining stellar models of different initial masses (and other initial parameters such as composition) to make a prediction of how an entire stellar population would appear at various ages. This would be similar to a star cluster. In contrast a mixture of stellar populations with a range of ages would simulate the stellar population of a galaxy.

The general result we find from population synthesis is that most stars are found on the main-sequence when they fuse hydrogen to helium. We then expect stars to evolve into red supergiants, white dwarfs and the other stellar types depending on their initial mass, composition and mass loss. We find that the luminosity of the brightest stars on the main sequence depends on the age of the cluster as the most luminous stars are the first to evolve off the main-sequence.

This simple picture is complicated by binaries. Binary interactions can prevent certain evolutionary pathways; for example, preventing stars from becoming red giants by removing their hydrogen envelopes. However this opens up alternative pathways not available to single stars. Recent work has revealed the importance of binary evolution in accurately modelling stars and stellar populations [3]. Rather than becoming red giants, the exposed cores evolve to become helium-rich dwarfs with significantly higher surface temperatures. When one star loses its hydrogen envelope the companion star can accrete the material, increasing the mass of the star. In extreme cases the two stars can merge. These processes can upset the simple relationship between the most massive and luminous stars and the age of a stellar population.

Adding to this complexity, new evolutionary pathways introduce greater variation due to an increased number of initial parameters. In binary systems we must account for the mass of both stars and their initial separation. These will sensitively determine when the stars will interact and the severity of this interaction. Finally the physics of binary evolution is more uncertain than single star evolution because it is inherently more complex and based on short-timescale dynamical processes. 
In this chapter we outline these aspects of population synthesis in more detail and pay special attention to uncertainties and complexities that are still being uncovered. We then discuss some recent and interesting observational constraints concerning supernova. These include their relative rates, their delay-time distribution, observed supernova progenitors and the detection of merging compact remnants.

\section{Stellar evolution}

Even though the Sun and other stars appear to be unchanging on human timescales the process of nuclear fusion at their centre is slowly using up their energy supply. Over millions and billions of years stars continue to change and evolve until they use up all their nuclear fuel. Before describing how a population of stars is expected to evolve, we must first review the basics of how stars evolve.

\subsection{Single stars}

Stars are spherical fluid objects in hydrostatic equilibrium. Their cores are hot enough to undergo nuclear fusion. All stars initially burn hydrogen to helium and then evolve off the main sequence and burn successively heavier elements in their cores; the more massive the star the heavier the elements it is able to fuse. Low-mass stars form only a helium core. Intermediate-mass stars can fuse helium to form a carbon-oxygen core. Massive stars can fuse up to a core of iron-group elements.

For low and intermediate mass stars, once these cores are formed their evolution is normally over; their hydrogen envelopes will be lost and those cores go on to become white dwarfs. The massive stars that have formed iron cores will explode in a core-collapse supernova typically leaving a neutron star or black hole remnant.

While burning hydrogen, stars are normally small and compact main-sequence stars. They grow in radius to become red giants once a helium core is formed. One aspect that greatly affects the evolution of stars is mass loss in stellar winds. Only massive stars, or other stars near the end of their lives, have strong stellar winds that can effect the evolution of a star. The evolutionary pathway taken for single stars is a competition between the progression of nuclear burning at the centre of the star and mass loss from the surface.

For low and intermediate mass stars, mass loss becomes strong when the star becomes a large red giant. These stars have a helium or carbon-oxygen core and the envelope loss results in a white dwarf. For massive stars, mass loss has the key impact on determining the nature of their observed supernova. For example if mass loss is weak then the supernova will be hydrogen-rich (type II). When the winds are strong enough to remove the hydrogen and leave only the helium core, the supernova would be hydrogen-poor (type Ib/c). We summarise the expected evolutionary pathways and their corresponding mass ranges at Solar metallicity in Table 1 


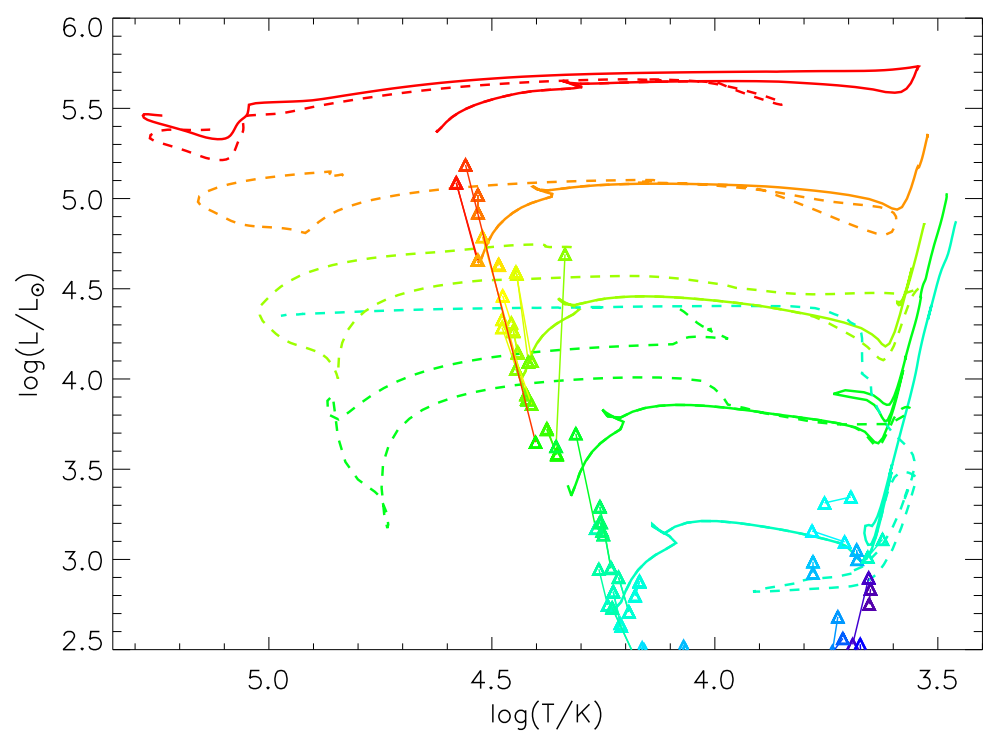

Fig. 1 An example Hertzsprung-Russell diagram showing massive star evolution tracks and observed eclipsing binary stars for which mass, temperature and luminosity can be accurately determined [2]. The shading represents the mass of the stars, solid line tracks are for single stars and dashed tracks are for binary stars. The masses of the tracks are 5, 7.5, 12, 20 and $40 \mathrm{M}_{\odot}$.

The values in Table 1 vary for stars of different initial metallicities or rotation rates. Changes are more significant for massive stars that will go to core-collapse. If a star is rotating fast enough to induce a strong mixing effect it forms a larger core and evolves as a slightly more massive star. This effect will be biggest in reducing the initial mass for type $\mathrm{Ib} / \mathrm{c}$ supernovae. Typically these differences are only a few $M_{\odot}$. The differences can be much larger when metallicity is varied. For example, if the metallicity is decreased this leads to weaker stellar winds that have the opposite effect and so will increase the minimum mass for a type $\mathrm{Ib} / \mathrm{c}$ supernova from a non-rotating star to an initial mass of $80 M_{\odot}$ at $1 / 10$ th Solar metallicity.

We show some sample evolution tracks on a Hertzsprung-Russell diagram in Figure 1 in which a star's luminosity and surface temperature are related. On Figure 11we include stellar models and observed eclipsing binary stars (where the parameters are well known) for comparison. We can see many of the stars are on the main sequence. Relatively few post-main sequence binaries are known because the postmain sequence lifetime is short and the stars in binaries are more likely to have interacted. This simple diagram demonstrates that we can have confidence in stellar models as they reproduce at the same time the observed masses, luminosities and surface temperatures. 


\subsection{Binary stars}

A binary star system is one in which two stars are bound together by their mutual gravity, orbiting around their common centre of mass. The mass, period and orbital separation are related by Kepler's Third Law which can be expressed as,

$$
\frac{\left(M_{1}+M_{2}\right)}{M_{\odot}}=\frac{(a / \text { A.U. })^{3}}{(P / \mathrm{yr})^{2}}=\frac{\left(a / 215 \mathrm{R}_{\odot}\right)^{3}}{(P / 365.25 \text { days })^{2}} ;
$$

where $M_{1}$ and $M_{2}$ are the masses of the two stars in the binary, $P$ is the orbital period in years or days and $a$ is the orbital separation in astronomical units or Solar radii.

For a star in a binary there is the possibility of extra physical processes. For example, tidal forces exchanging angular momentum between the stars' rotation and their orbit. This effect may be enhanced if magnetic fields are present in the

Table 1 Mass ranges for typical evolution of single stars, along with nuclear burning reactions during each of the evolutionary states. The different phases are the main sequence (MS) when stars are hydrogen burning the other types occur post-main sequence: red giant branch (RGB), asymptotic giant branch (AGB), super-AGB (SAGB), red supergiants (RSG), Wolf-Rayet (WR) stars, blue supergiants (BSG) and luminous-blue variables (LBV). We also include two types of supernovae (SNe) here, type II with hydrogen and type Ib/c without hydrogen in the spectra. The remnants are either a helium white dwarf (HeWD), a carbon-oxygen white dwarf (COWD), a oxygen-neon white dwarf (ONeWD), a neutron star (NS) or a black holes (BH). The values are for Solar metallicity and the values due vary with metallicity, initial rotation rate and binary interactions as discussed in the text.

\begin{tabular}{|c|c|c|}
\hline$M_{\text {initial }} / M_{\odot}$ & Evolution pathway & Total Lifetime \\
\hline$<0.08$ & $\begin{array}{l}\text { Mass at centre of star too low for nuclear fusion, the object is a } \\
\text { brown dwarf rather than a star. }\end{array}$ & \\
\hline $0.08-0.8$ & $\begin{array}{l}\mathrm{MS} \rightarrow \mathrm{RGB} \rightarrow \text { HeWD } \\
\text { H-burning only } \rightarrow \text { Strong stellar-wind removes envelope. }\end{array}$ & 4500-29 Gyrs \\
\hline $0.8-6.5$ & $\begin{array}{l}\mathrm{MS} \rightarrow \mathrm{RGB} \rightarrow \mathrm{AGB} \rightarrow \mathrm{COWD} \\
\mathrm{H} \text {-burning } \rightarrow \text { He-burning } \rightarrow \text { Strong stellar-wind removes enve- } \\
\text { lope. }\end{array}$ & 29Gyrs-66Myrs \\
\hline $7-8$ & $\begin{array}{l}\text { MS } \rightarrow \text { RGB } \rightarrow \text { AGB } \rightarrow \text { SAGB } \rightarrow \text { ONeWD } \\
\text { H-burning } \rightarrow \text { He-burning } \rightarrow \text { C-burning } \rightarrow \text { Strong stellar-wind re- } \\
\text { moves envelope. }\end{array}$ & $66-41$ Myrs \\
\hline $8-20$ & $\begin{array}{l}\mathrm{MS} \rightarrow \mathrm{RSG} \rightarrow \text { Type-II SN } \rightarrow \text { NS } \\
\mathrm{H} \text {-burning } \rightarrow \text { Fusion progresses to an iron-group elements core. }\end{array}$ & 41-9.7 Myrs \\
\hline $20-25$ & $\begin{array}{l}\mathrm{MS} \rightarrow \mathrm{RSG} \rightarrow \text { Type-II SN } \rightarrow \mathrm{BH} \\
\mathrm{H} \text {-burning } \rightarrow \text { Fusion progresses to an iron-group elements core. }\end{array}$ & 9.7-7.7 Myrs \\
\hline $25-40$ & $\begin{array}{l}\mathrm{MS} \rightarrow \mathrm{RSG} \rightarrow \mathrm{WR} \rightarrow \text { Type-Ib/c SN } \rightarrow \mathrm{BH} \\
\mathrm{H} \text {-burning } \rightarrow \text { Fusion progresses to an iron-group elements core } \\
\rightarrow \text { Strong stellar-wind removes envelope. }\end{array}$ & 7.7-5.1 Myrs \\
\hline $40-100$ & $\begin{array}{l}\mathrm{MS} \rightarrow \mathrm{BSG} / \mathrm{LBV} \rightarrow \mathrm{WR} \rightarrow \text { Type-Ib/c SN } \rightarrow \mathrm{BH} \\
\mathrm{H} \text {-burning } \rightarrow \text { Fusion progresses to an iron-group elements core } \\
\rightarrow \text { Strong stellar-wind removes envelope.. }\end{array}$ & $5.1-3.1$ Myrs \\
\hline
\end{tabular}


stars. However the most important extra process is the possibility of mass transfer that leads to both enhanced mass loss and mass gain for the stars in a binary.

Using the mass ranges in Table 1 as a guide, enhanced mass loss in binary systems means that any star initially more massive than $10 M_{\odot}$ can now lose its hydrogen envelope and give rise to a type $\mathrm{Ib} / \mathrm{c}$ supernova. While mass gain by stars means that, for example in an extreme case, a binary with two $10 M_{\odot}$ stars could merge and form a $20 M_{\odot}$ star. This would happen at ages up to $25 \mathrm{Myrs}$, the main-sequence lifetime of the $10 M_{\odot}$ stars. Therefore the $20 M_{\odot}$ might appear to be surrounded by an older population than its typical 9.7Myrs lifetime. Many clusters have such stars in the population as blue stragglers.

In the simplest and extreme view, one can expect that if a star's radius grows to be of the order of the orbital radius the stars will "get in each other's way". In reality the interaction happens before a star grows to the size of the orbit. In the rotating frame of the binary, along the line between the centre of the two stars there is a point where the gravity of the two stars is equal. If stellar material of one star goes beyond this point, the other star's gravity will dominate and pull the material towards it. The surface where the gravity of the two stars cancels is called the Roche Lobe. Nearly every stellar evolution code assumes spherical symmetry, so approximate expressions are calculated to estimate the radius, $R_{\mathrm{L}}$, at which a star fills its Roche Lobe. The most widely used one was derived by Eggleton [4],

$$
R_{\mathrm{L}, 1} / R_{\odot}=\frac{0.49 q^{2 / 3}}{0.6 q^{2 / 3}+\ln \left(1+q^{1 / 3}\right)},
$$

where $q=M_{1} / M_{2}$ is the mass ratio of the two stars. If a star fills this volume it can be assumed that any mass beyond this volume flows from the star to its companion. This is referred to as Roche-Lobe Overflow (RLOF) and leads to both mass loss and mass gain for the primary and secondary stars respectively. This mass exchange can be considerably stronger than the typical mass loss driven by stellar winds.

During this mass transfer angular momentum is also transferred. This can lead to the companion star being spun up to almost critical rotation rates[5]. In fact the most rapidly rotating stars are likely to be the result of binary evolution. Those stars that are spun up can experience evolutionary pathways that could lead to long-GRBs if they also become massive enough[6].

If RLOF does not prevent the radius of the overflowing star from growing it may eventually engulf the other, this leads to common-envelope evolution (CEE). Here the core of one star and its companion now orbit around one another within the envelope of the larger star. The exact details of such evolution are extremely uncertain. We know it must occur because of the large number of white dwarfs in very close binary systems with periods of days. These are only possible if the system's excess angular momentum has been carried away by an ejected common envelope. Our theoretical understanding of CEE is still at its beginning[7]. We do understand that there are two possible outcomes from CEE, either for the two stars to merge or for the formation of a much tighter binary, with one of the two stars having lost its hydrogen envelope. 
In comparison to CEE, RLOF is relatively well understood as there are a number of observed systems where this process is occurring. One of the better known systems for example is $\beta$-Lyrae where stable mass-transfer is still happening today at the rate of approximately $2 \times 10^{-5} M_{\odot} \mathrm{yr}^{-1}[8]$.

The key issues of mass transfer in population synthesis concern whether a binary systems only experiences RLOF or whether the interaction progresses to CEE. CEE can only be avoided if mass loss can prevent the star from expanding and also if mass transfer causes the orbit to shrink or grow. Typically if the more massive star is the donor then the orbit shrinks on mass transfer which can lead to CEE. However if the transfer is not fully conservative and mass is lost the system can be prevented from shrinking and entering CEE. If the less massive star is the donor the orbit widens.

The evolutionary state of a star can also affect mass transfer stability. Typically mass transfer during the main-sequence is driven at a nuclear evolutionary timescale so mass transfer is slow and stable. For post-main sequence mass transfer the evolutionary timescale is shorter so CEE becomes more likely. We note that tidal forces at the time of interactions can also be important. As one star grows it can be spun up at the expense of angular momentum from the orbit, again possibly instigating CEE. Many of these factors are relatively well understood. The one that is uncertain and most important is how efficient or conservative mass-transfer is. It appears that while, in general, mass-transfer is efficient between stars with similar initial masses there are more complex factors at work that we still need to understand [9, 10].

While we have limited understanding of CEE it is typically parametrised in population synthesis[7, 11] by comparing the binding energy of the overflowing star's envelope to the orbital energy of the core of the primary star and its companion,

$$
E_{\text {bind }, \mathrm{i}}=\alpha_{\mathrm{CE}}\left(E_{\mathrm{orb}, \mathrm{f}}-E_{\mathrm{orb}, \mathrm{i}}\right) .
$$

Where,

$$
E_{\mathrm{bind}, \mathrm{i}}=\frac{-G M_{1} M_{\mathrm{env}, 1}}{\lambda R_{1}}
$$

is the initial binding energy of the envelope of the star with $\lambda$ being a constant depending on the structure of the star and $M_{\text {env, } 1}$ being the mass in the envelope of the donor star. Then,

$$
E_{\text {orb }}=\frac{-G M_{c, 1} M_{2}}{2 a}
$$

is the initial or final orbital energy of the primary's core of mass $M_{\mathrm{c}, 1}$ and the secondary star. The free-parameter in the model is $\alpha_{\mathrm{CE}}$ and is a constant of the order 1. While there are subtle variations on this model they all effectively use conservation of energy. Although a model including angular momentum of the stars and orbit has also been considered[12]. Of course if there is a significant amount of binding energy then CEE can lead to the merging of the two stars.

In Figure 1 we show examples of evolutionary tracks of stars that experience RLOF or CEE compared to the single star tracks. The main result is that the stars lose their hydrogen envelope and undergo further evolution as helium stars or Wolf- 
Rayet stars. The greatest difference compared to the single-star evolution in Figure 1 and Table 1 is for stars between 8 to $25 M_{\odot}$. For these stars stellar winds are not strong enough to remove the hydrogen envelope at any stage of evolution. Therefore only RLOF or CEE can remove the hydrogen envelope to create helium stars. Stars in the other mass ranges might have their mass loss accelerated but the eventual outcome is not strongly affected.

Typically in a binary system the initially more massive star evolves faster and hence is the first to interact. Whether the lower mass companion also experiences RLOF or CEE depends on whether the binary is disrupted or not. A binary can be disrupted if, for circular orbits, half of the total mass of the binary is ejected from the system over a short timescale. For low and intermediate-mass stars this can be unlikely. But in massive stars a supernova can rapidly eject a large amount of mass and can unbind the binary.

The loss of half the mass in a binary leads to the end of the binary, as the system does not have enough mass for gravity to hold it together. The compact remnant and the companion star will therefore fly apart and continue their evolution as single stars. The extreme cases in this scenario are for type Ia or pair-instability supernovae where the exploding star is disrupted. In these cases one star is totally destroyed, leaving the other alone.

The complicating factor in this picture is that there is strong observational evidence that, in supernovae, neutron stars and black holes receive kicks when they are born [13, 14]. The exact reason for the kick is likely due to asymmetries during core-collapse and the subsequent explosion. We know that some neutron stars can have velocities up to $1000 \mathrm{~km} \mathrm{~s}^{-1}$, although the distribution is similar to that of a Maxwell-Boltzmann distribution with a characteristic velocity of $265 \mathrm{~km} \mathrm{~s}^{-1}$. For black holes there is less direct evidence of how strong the kicks should be but it is probable that their kicks are weaker [15].

These supernova kicks can either unbind a binary that would have remained bound or ensure a binary remains bound rather than unbound. Of course this depends sensitively on the strength of the kick and the direction. When the binary remains bound further evolution can lead to binary interactions between the secondary star and the primary star's compact remnant. These systems are observed as X-ray binaries. To first order the physics of these systems is similar to that of binaries with two normal stars. The two important differences are that the compact remnants tend to accrete smaller amounts of material and are luminous in X-rays. These lead to more stable mass transfer and the irradience of the companion star affecting mass transfer respectively. The main uncertainty is how much mass can be accreted because that determines the efficiency of mass transfer and whether CEE happens in these binaries or not. In such systems a second supernova can also occur giving rise to either two runaway compact remnants or a double compact remnant binary that may be observed as gravitational wave sources if they ultimately merge.

Because the magnitude and direction of kicks are usually assumed to be random with respect to the position of the exploding star, multiple kicks must be simulated with each binary model to predict the full possible range of possible outcomes from a supernova. This is one of the reasons why population synthesis was created - the 
evolution of a binary is no longer linear and single valued. An element of randomness enters when a supernova occurs. This is again another reason why so many computations are required in binary population synthesis as all the possible evolutionary avenues must be explored and accounted for statistically.

\section{Making a synthetic stellar population}

While individual stellar models are useful and can be used to constrain our understanding of stellar evolution the next step is to combine these models together to predict the appearance of a population of stars. First we discuss how to do this with single star models and then explain how it becomes more complex with a binary population.

\subsection{Single stars}

Using single-star models, population synthesis is relatively straightforward. We take stellar evolution tracks as shown in Figure 1, which demonstrate how a star of a single mass evolves over time, and combine them in proportions given by an estimated distribution of initial masses to indicate how stars of different masses look at the same age. We show such a combination for single stars in Figure 2 In the Figure we illustrate how synthetic populations appear at different ages and compare them to observed clusters. The contours represent the probability of stars being observed in a certain region of the Hertzsprung-Russell diagram. We can see that as the population ages we eventually find red (super)giants appearing and the main sequence shortens, with the most massive stars evolving faster than the less massive stars. Matching models to the distribution of turn-off and post main-sequence stars in individual clusters, we find that the age of Cygnus OB is closest to 3Myrs and Upper Scorpius 10Myrs. We note that normally for single stars isochrones are created which are lines of constant age but varying mass. Here we have calculated density/contour plots so we can later compare them to binary models where a simple isochrone is not possible.

To create such plots from the stellar models, the appearance of all possible stellar models at a specific age are recorded and any gaps in the model mass distribution are interpolated over. This is less simple than it sounds. One problem is the vast range of rates of evolution for stars of different masses. For example a $100 M_{\odot}$ star will evolve over $3 \mathrm{Myrs}$ compared to a $1 M_{\odot}$ star's lifetime of $10 \mathrm{Gyrs}$. The postmain sequence lifetime of such stars might be only $10 \%$ of their total lifetime which makes it difficult to account for the shortest phases of evolution. Therefore either care must be taken in interpolation between the different stellar models or some binning of the models must be used as in Figure 2 . Here we use time bins of width $0.1 \mathrm{dex}$ in the logarithmic age of the stellar population. 
The second problem we must deal with is that we must account for how many stars there are of each mass. This is typically referred to as the initial-mass function (IMF) which describes how many stars there are of different masses. The most basic form was first derived by Salpeter [18] who proposed a simple power-law,

$$
\frac{d N}{d M} \propto M^{-2.35}
$$

where $N$ is the number of stars of mass $M$. Much work has been performed over the last few decades to determine the IMF. The most commonly used one now is by Kroupa [19]. It is similar to Salpeter at high mass but becomes shallower below $0.5 M_{\odot}$ to such that $\frac{d N}{d M} \propto M^{-1.35}$ down to a minimum mass of $0.1 M_{\odot}$. The IMF does also continue down to lower masses but this is the domain of brown dwarfs and planets. There are also other forms [20], but all tend to have the same high mass slope but vary at the lower masses.

Both the IMF and the longer evolutionary times imply that there are many more low-mass stars than high-mass stars. This compounds the problems with interpolation as the fainter coolest stars are the most numerous but the rarest, brightest stars dominate the appearance of a cluster when it is young. There are solutions to these problems. The interpolation and careful selection of the initial masses of the stellar models used to create the populations are key.

For a simple demonstration of how important this is we can combine the above IMF with an approximation of how the lifetime of a star depends on mass. From homology we can prove that for most main-sequence stars with radiative interiors, supported by gas pressure, $L \propto M^{3}$ and so the main sequence lifetime is given by, $\tau \propto M^{-2}$. Therefore incorporating this equation with the IMF we find that in a stellar populations that continuously forms stars the number of stars at a given mass will

be $\propto M^{-4.35}$. However given that the more massive stars are more luminous, the contribution of stars to the luminous output of a galaxy is not quite so skewed to the lowest mass stars. The luminosity contribution of a star is $\propto M^{-1.35}$ assuming continuous star formation and $\propto M^{0.65}$ for a very young star cluster.

For a single star population other parameters that can be varied are initial composition, rotation rate and the scheme of mass-loss rates employed when the stellar models are calculated. For example the tracks in Figure 1 and 2 are assumed to be Solar metallicity which is taken to be $Z=0.02$ (although there is some debate on exactly what Solar metallicity is [21]). This just determines which generation of stars the models represent. If $Z=0$ they would be the first generation of stars in the Universe. The amount of hydrogen in the models would also vary from $X=0.70$ to 0.75 respectively. The remaining amount of mass in the stars will be made up of helium.

Changing the metallicity has strong effects on the opacity of the stellar material and thus the stellar radii. Crucially, it also affects the mass-loss rates in stellar winds. Most winds are driven by radiation pressure from the iron lines in the atmosphere. Therefore higher metallicities have more iron which subsequently leads to stronger stellar winds. Stellar winds become strongest for the most luminous stars and generally have the dramatic effect of removing a star's hydrogen envelope. For low- and 


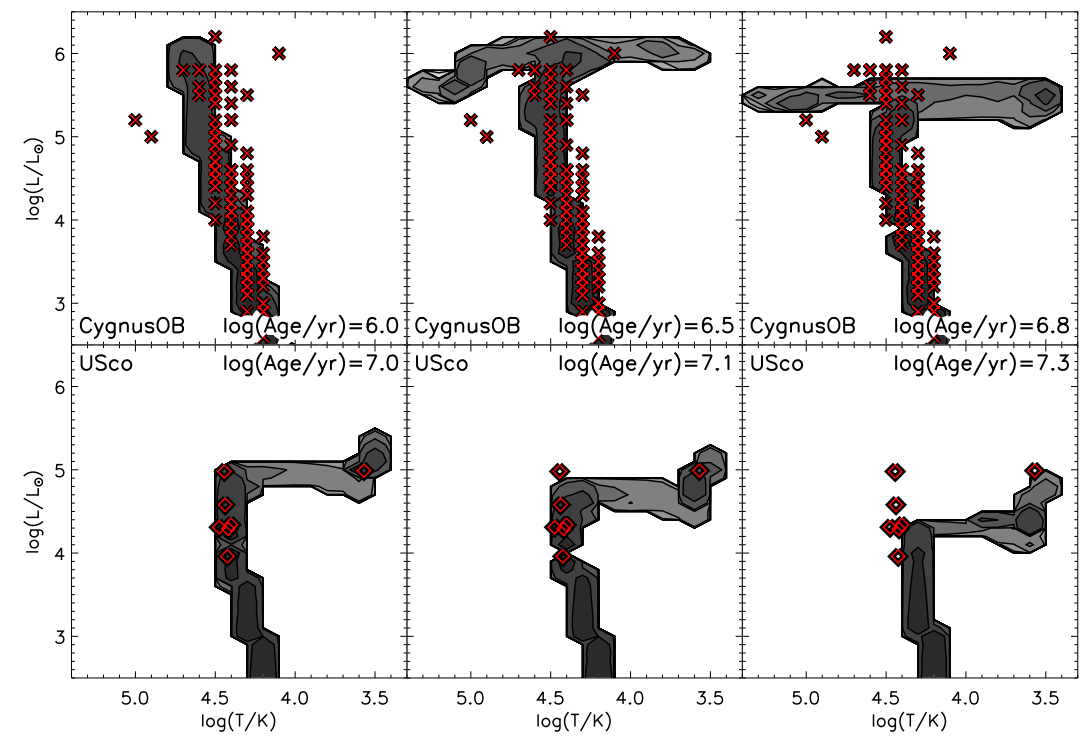

Fig. 2 Example Hertzsprung-Russell diagrams showing a synthetic single-star population compared to the observed populations of two stars clusters, the Cygnus OB association[16] in the upper panels in crosses and USco association [17] in the low panels in diamonds. The age of the population can be estimated by comparing the distribution of the most luminous stars. Each contour is separated by an order of magnitude in the probability density of stars on the HR diagram.

intermediate-mass stars this leads to RGB and AGB stars which later become white dwarfs. For the massive stars it leads to hydrogen-poor Wolf-Rayet stars.

Unfortunately the mass-loss rates employed in a stellar model are mostly empirical with only a few based on theoretical calculations. Hence some changes can be made in the predictions of stellar models by altering the mass-loss rates assumed in the stellar models. By comparing to observations we can constrain which rates best fit the observation. Similarly, varying the rotation rate does change the evolution of stars by extending their main-sequence lifetimes. Also the stars tend to have higher surface temperatures as they evolve. Therefore there are changes to the shape of the populations on the HR diagram as they age. [22].

\subsection{Binary stars}

All the details that are required to make a single-star synthetic population also apply for a binary population. However now we need to worry about more than just the IMF. There must also be an initial distribution for how many binaries there are, as well as the orbit and the mass of the second star. The simplest assumption is that 


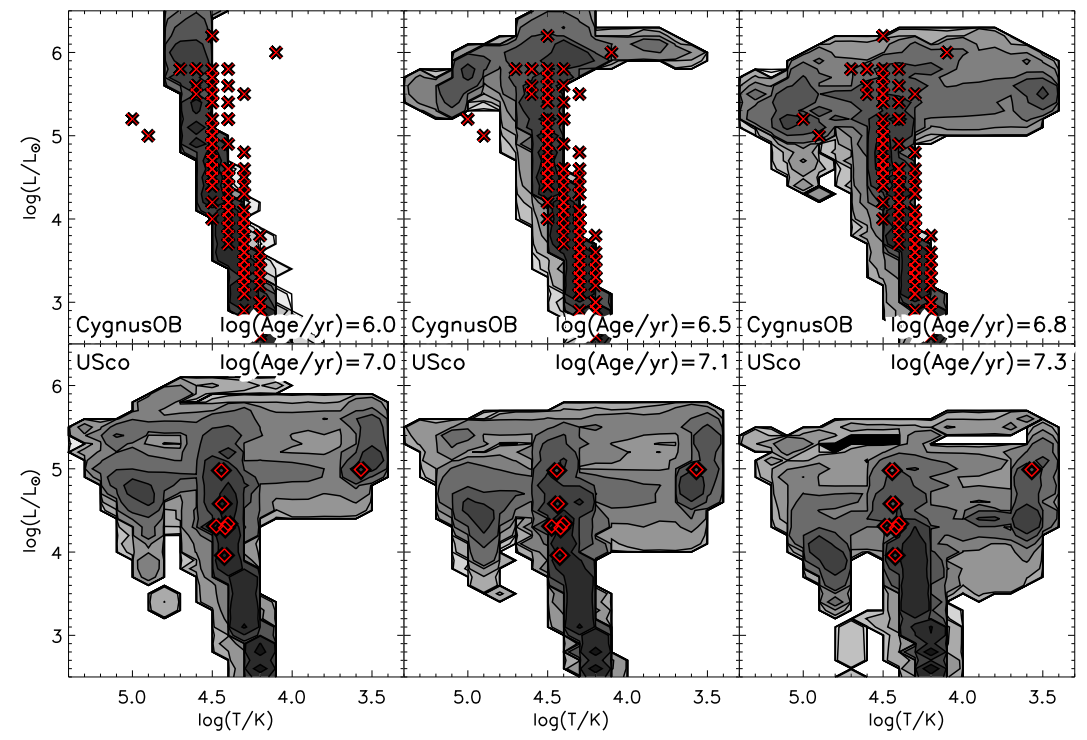

Fig. 3 Similar to Figure2 but now with a synthetic binary star population compared to the observed populations of two star clusters, the Cygnus OB association[16] in the upper panels and USco association [17] in the lower panels. The age of the population can be estimated by comparing the distribution of the most luminous stars but is more uncertain in the case of binary populations. Each contour is separated by an order of magnitude in the probability density of stars on the HR diagram.

there is a distribution in the mass ratio of the secondary to the primary star. This is normally defined as $q=M_{2} / M_{1}$ and is assumed to range from 0 to 1 with a distribution of $f(q)=q^{\gamma}$, typically with $\gamma=0$. The second detail required is orbital separation or period distribution. The simplest assumption here is Öpik's law which assumes a flat distribution in $\log P$. Yet the number of binaries in the population has no real theoretical basis and must be measured.

For many years it has been difficult to measure these parameters. The current understanding is that for massive stars above $8 M_{\odot} 60 \%$ are in multiple systems, while above $16 M_{\odot}$ this increases to $80 \%$. Furthermore a small number of these multiples include more than one companion star. In comparison only a quarter of the lowest mass stars are in multiple systems [23]. For most mass ranges $\gamma$ is consistent with being flat. For massive stars $\gamma=-0.1 \pm 0.6$ although for systems wider than $100 \mathrm{AU}$ we find that $\gamma=-0.5 \pm 0.1$ indicating a preference for more unequal mass ratios. The period distribution is also consistent with Öpik's law although there is also a slight excess of systems with 5 day periods in this distribution [23]. Other mass ranges tend to have typical orbital separations with smaller orbits for lower mass stars. 
Finally the eccentricity distribution is slightly biased towards circular orbits with a range of eccentricities up to close to 1 . Approximately circular systems are more likely and a recent estimate is that, $d N / d e \approx 0.4+1.2 e$ [24]. It is uncertain how important the eccentricity is for the evolution of binary systems. It has been suggested that tides circularise the orbits before interactions [11]. Thus the semi-latus rectum distribution is the most important initial parameter. Conversely in some cases eccentricity during mass transfer may be important for determining the outcome of the mass transfer [25]. It is clear, that closer binaries are less likely to be eccentric than the wider binaries that represent the effect of tides circularising the binary.

In light of knowing these distributions we are now left with the question of how to sample this parameter space with stellar evolution models. There are the two widely used techniques of creating binary stellar models, rapid and detailed models. For the rapid method a full sampling of the initial parameter space is possible. The initial masses, periods and eccentricities can be sampled at random or uniformly from the input distributions. The rapid models can be calculated in a fraction of a second, thus many models could be calculated to simulate a population. These models necessarily make approximations and apply analytic prescriptions for some aspects of stellar evolution [26, 11, 27].

Only recently it is possible to calculate large numbers of detailed models, where the full stellar structure is computed, due to the increase of computational resources. A detailed model grid was first calculated by the Brussels group [28]. Now more groups are also taking up this method [29, 30] gaining accuracy in the models of stellar structure and evolution. However because models take minutes to run the computational cost is a few orders of magnitudes greater than the rapid models. Thus their ability to search the parameter space is severely constrained. It is therefore important for the detailed models to sample the space over a uniform grid.

One further detail must be accounted for during the evolution of the binary system: what happens after the first (and second!) supernova. The neutron star kick can destroy the binary or keep it bound. The kicks are modelled as discussed above but the full range of directions and kick strengths must be sampled. While the kicks are uncertain, increasing accuracy in supernova simulations and observations provide growing evidence that neutron star kicks may be less random than currently suggested [31, 32]. Black hole kicks are a little more uncertain, mainly due to the lack of observations of single black holes. Many seem to suggest that there are at least weak kicks [15].

Creating detailed models after the first supernova is tricky due to the large number of possible outcomes for each individual model. This is when the rapid evolution models have the advantage because it is possible to sample all the probable future evolutionary pathways in the same time it takes to blink. It is also possible to investigate the effect of all the uncertainties, such as initial parameters, common-envelope evolution model and supernova kick distributions. This provides an understanding of where we should focus our efforts on reducing those uncertainties.

Once the models are calculated, again they must be processed to predict the details of the synthetic population for comparison to observations. A similar method can be used as before and we plot in Figure 3 a new version of Figure 2 but for a 
binary population. We see the predictions change and fill a larger parameter space. There is no longer a single isochrone for a single-aged population. The processes of RLOF and CEE cause some of the stars to evolve on different paths. Importantly we see that the derived ages become slightly different for the binary population. In general binary populations appear bluer and more luminous at the same age than single-star populations. This suggests at least for Cygnus OB the age could be up to $6 \mathrm{Myrs}$ rather than the $3 \mathrm{Myrs}$ suggested by single star models.

The key evidence that binaries are important for population synthesis come from the direct observational evidence from X-ray binaries, double neutron-star binaries and gravitational waves from black-hole mergers. The observation of such systems indicates that many supernovae must also happen within binary systems and there is growing observational evidence of this from supernovae themselves.

\section{Binary population synthesis of supernovae and their progenitors}

In this section we highlight the inferences that are important for understanding supernovae from population synthesis.

\subsection{Type Ia thermonuclear supernovae}

From the early stages of supernova studies it was realised that some of the events with no hydrogen arose from a different stellar population [33]. It was an old rather than young stellar population indicating that the progenitors must have been a few 100 Myrs or older. Eventually it was realised that the explosion of a white dwarf at the Chandrasekhar limit could explain the energy released in the supernovae and explain the uniformity of the explosions.

A white dwarf is the burnt out remnant of an intermediate mass star within which no further nuclear fusion reactions are possible. The objects are supported by electron-degeneracy pressure. It was Subrahmanyan Chandrasekhar who first combined special relativity and quantum mechanics to show that there was a maximum mass for a white dwarf. When the mass reaches or exceeds this limit the star cannot be supported and collapses. In the case of a carbon-oxygen white dwarf this can lead to the ignition of explosive nuclear fusion of carbon followed by a thermonuclear supernova [35, 36, 37].

We mention these as they are an important result of binary evolution and may provide firm constraints in the future. There is also some overlap between what might be type Ia and core-collapse events. For example the type Iax events have been suggested to be either core-collapse or thermonuclear events. For these events a possible progenitor has been detected. Supernova $2012 \mathrm{Z}$ had its progenitor ob- 


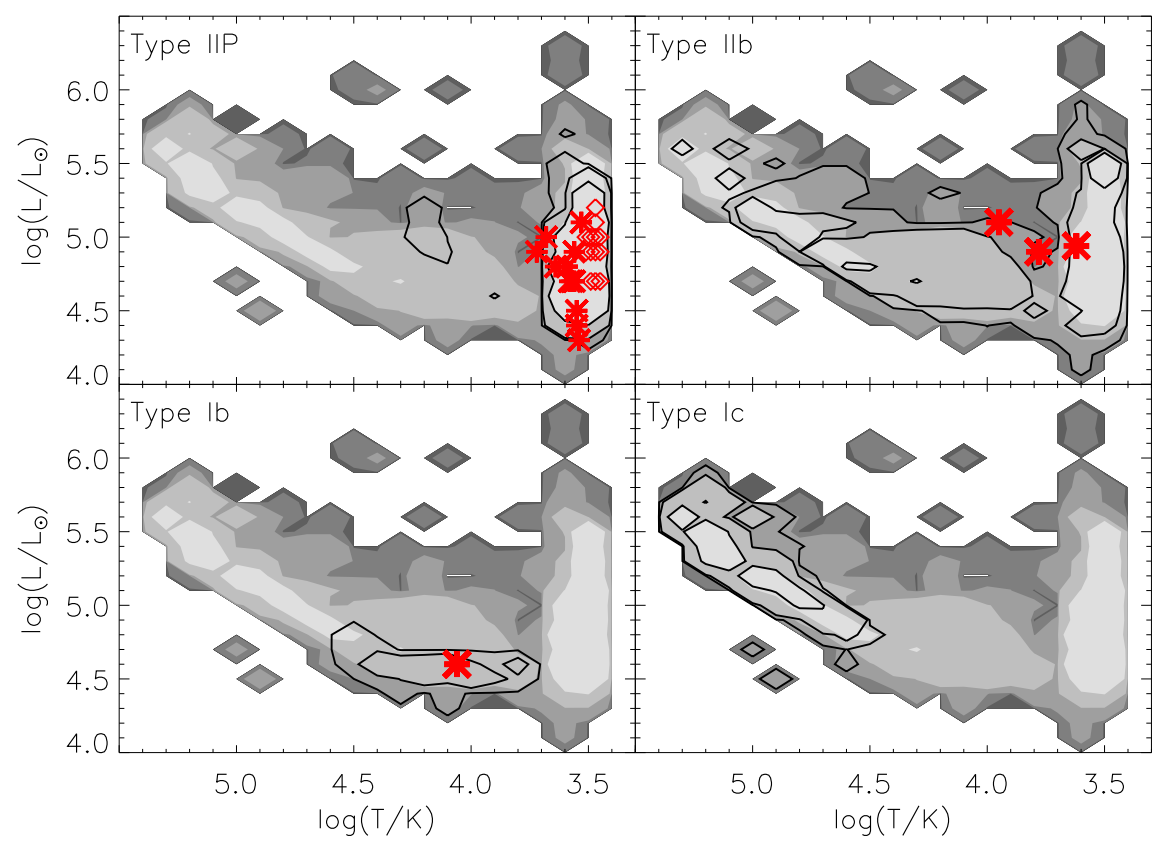

Fig. 4 Example Hertzsprung-Russell diagrams with the predicted location of supernova progenitors compared to observed progenitors 34]. Grey contours are for all progenitors while the black contours represent the location of a specific supernova type as labelled in each panel. The asterisks in each panel represent the location of observed progenitors and the diamonds are upper limits.

served as a faint blue star [38] suggesting the progenitors are possibly helium stars transferring matter onto an exploding white dwarf.

\subsection{Core-collapse supernovae}

The more common type of supernovae arises from the final core-collapse of massive stars. These are stars that are initially more massive than $8 M_{\odot}$ and in which nuclear fusion progresses all the way to form an iron-group element core. Further nuclear fusion releases no energy so the core collapses to a neutron star or black hole depending on the mass of the core. This collapses releases a tremendous amount of energy that causes the star to explode. While this general picture holds, some of the exact details are still being worked out. Only recently supernova simulations have been able to lead to model explosions.

The core-collapse supernovae fall into two principal types, type II and type Ib/c which are hydrogen-rich and hydrogen-free respectively. Population synthesis' key role is in predicting the relative populations of these two supernovae as they depend primarily on the amount of mass loss the progenitor stars experiences before they 
explode. For single stars this is dependent on their stellar winds, while for binary stars it is the result of CEE and RLOF.

There is a strong consensus from multiple authors that interacting binaries must dominate the rate of observed core-collapse supernovae [29, 39, 40, 41, 42, 43]. All these studies show that binaries increase the number of hydrogen-free progenitors at the expense of red supergiant, hydrogen-rich progenitors. This is a common feature of nearly all binary population predictions concerning core-collapse supernovae.

One important feature that population synthesis can predict is how the observed ratio of supernova types varies with metallicity. It was initially thought that the relative SN rates should be constant with binary interactions but vary for single stars because stellar winds are radiatively driven and thus metallicity dependent. The fact is that binary interactions only remove some, not all, of the hydrogen on the surface of a star and the rest must be removed by stellar winds. This leads to more type II $\mathrm{SN}$ relative to type $\mathrm{Ib} / \mathrm{c} \mathrm{SNe}$ at lower metallicities.

While the rates provide an important constraint on binary stellar population synthesis, a more direct indication is the growing number of observed progenitors of core-collapse SNe. The next step for population synthesis is to model the distribution of progenitors in the HR diagram as well as the relative rates of the different types of SNe. We show in Figure 4 the predicted location of the different corecollapse SNe compared to the locations of observed SN progenitors. The most commonly detected progenitors are the type II events while only one type Ib supernova progenitor is detected. The detected progenitors also show surface temperatures of below $10,000 \mathrm{~K}$; likely because hotter stars output most of their flux in the ultraviolet and are also more affected by dust which make them difficult to detect in pre-explosion images [43, 44].

Studying the progenitors has revealed that the type II supernovae lack the most luminous and massive predicted progenitors. In comparison the progenitors of type $\mathrm{Ib} / \mathrm{c}$ supernovae all remained unobserved despite the expected progenitors, WolfRayet stars, being some of the most luminous. One problem is that while they might have a high bolometric luminosity most light is emitted in the ultraviolet so they are also faint in the optical. A population synthesis model showed that the nondetections point towards most type $\mathrm{Ib} / \mathrm{c}$ actually came from binary progenitors. It was also suggested [44] that some of the progenitors from binaries, being cooler, should also be easier to observe. This was borne out by the detection of the first type Ib progenitor that (after a lot of competing studies) was shown to be most likely a helium giant evolved from a lower mass star [45, 46, 47, 48].

This leaves us with two unresolved questions, where are these predicted helium stars in our Galaxy and what happens to the Wolf-Rayet stars? Some of the WolfRayet stars may still explode but some may simply collapse directly to a black hole and have no supernova, as some of the more massive red supergiants have been suggested to [34]. Population synthesis cannot be used to resolve these questions yet as they depend too much on the uncertainties of CEE, RLOF and core-collapse itself.

One pathway that may be able to provide some answer to this question in future. A consistent result between binary population synthesis codes is that binary inter- 
actions extend the delay-time range for supernovae [49]. The delay time is the time between a star being formed and exploding in a supernova. In a single star population the range would be from the lifetime of a $100 M_{\odot}$ star to an $\approx 8 M_{\odot}$ star. Or between 3 Myrs to $41 \mathrm{Myrs}$. However for a binary population the interactions can lead to core-collapse supernova at ages up to 200Myrs after the stars were formed. This may not be a small effect with predictions of between $8.5 \%$ to $23 \%$ of supernova occurring after the single-star cut-off age. The exact number of "late" supernovae depends on the uncertainties in the population synthesis but all the codes to predict them. Future study of delay times will give us further evidence of the importance of binary interactions in supernovae but also possibly answer more about the nature of the progenitors for all supernova types.

Finally other observations that can be modelled and place constraints on synthetic populations involve modelling the outcomes of a binary in a supernovae. Much work for example has been done to model X-ray binaries [14, 15, 50], runaway stars and also long-gamma ray bursts that are associated with supernovae [6, 41, 51]. Combining multiple observations in future will provide greater insight into CEE and especially the creation of kicks for forming neutron stars and black holes.

\subsection{Compact remnant mergers}

Once the evolution driven by nuclear fusion is over for the stars in a binary the evolution becomes relatively simple. The stellar remnants, be they white dwarfs, neutron stars or black holes, are (to a good approximation) unchanging. This is somewhat untrue for white dwarfs as they do continue to cool, as do neutron stars to a smaller degree. The only remaining event to occur for such binaries is an eventual merger due to the tightening of the orbit by emission of gravitational radiation. The history of predicting mergers of compact remnant binaries is long.

Gravitational radiation is a natural consequence of the General Theory of Relativity and how the orbit and eccentricity of a binary were affected by gravitational waves were derived by Peters [52],

$$
\frac{d a}{d t}=-\frac{64 G^{3} M_{1} M_{2}\left(M_{1}+M_{2}\right)}{5 c^{5} a^{3}\left(1-e^{2}\right)^{7 / 2}}\left(1+\frac{73}{24} e^{2}+\frac{37}{96} e^{4}\right)
$$

and

$$
\frac{d e}{d t}=-\frac{304 e G^{3} M_{1} M_{2}\left(M_{1}+M_{2}\right)}{15 c^{5} a^{4}\left(1-e^{2}\right)^{5 / 2}}\left(1+\frac{121}{304} e^{2}\right)
$$

In the case of a circular orbit the time taken for a merger is given by,

$$
T_{\mathrm{m}, \mathrm{e}=0}(a)=\frac{5 c^{5} a^{4}}{256 G^{3} M_{1} M_{2}\left(M_{1}+M_{2}\right)}
$$

while for a highly eccentric binary with $e$ close to one, 


$$
T_{\mathrm{m}, \mathrm{e} \sim 1}(a, e)=\frac{768}{425} T_{\mathrm{m}, \mathrm{e}=0}\left(1-e^{2}\right)^{7 / 2}
$$

Using these equations it is straightforward to calculate how long it will take two compact remnants to merge. In many cases, due to the weakness of the gravitational radiation, the time to merge will be greater than the age of the Universe and so merger rates are relatively low, although interactions with a third body may increase the chance of a merger.

Before 2015 the only events that were observed and likely to be the result of such mergers were type Ia supernovae and short-GRBs, at least some of which are thought to be the result of white dwarf or neutron star mergers respectively. On the 14th of September 2015 the merger of two massive black holes was detected via the gravitational waves released during their final inspiral and merger [53]. This gave the final direct evidence that such mergers do occur. The fact that the first detection would be a binary black-hole merger was predicted by [54] and is no surprise considering that more massive black holes have a shorter merger time and also a stronger Chirp mass $\left(\mathscr{M}_{0}=\left(M_{1} M_{2}\right)^{3 / 5} /\left(M_{1}+M_{2}\right)^{1 / 5}\right)$. This makes them more likely to be detected as the the detection distance is $\propto \mathscr{M}_{0}^{5 / 6}$.

While the time it will take two compact objects to merge is straightforward to calculate given their masses and initial period, the prediction of the initial parameters of the compact remnant binaries is extremely uncertain. To predict it a stellar population must be modelled from birth to the final death-throes of both stars. Therefore every uncertainty in stellar evolution and population synthesis has an effect on the final merger population, as has any uncertainty in the star-formation history of the population. If the uncertainties of evolution are varied over a reasonable range, the final predicted results can span a large range [55] even though most models predict similar orders of merger rates [56, [57, 58, 30, 59]. Modelling the rate we would observe today from all compact remnant binaries formed in the Universe requires understanding of the mass and star-formation history of the Universe and how the metallicity of the Universe varies with the cosmic age [58].

The key problems are making enough close binaries with compact remnants and making enough massive black holes. For the remnants to merge within the age of the Universe they have to be massive, with their orbits highly eccentric and/or very close. In future as more merging compact remnants are detected the sensitivity of the models will be useful. As the population of observed mergers becomes understood there will be tighter constraints on the possible range of the input population synthesis parameters as well as possibly the star-formation history of the Universe. In addition it can be hoped that gravitational waves will be detected from neutron star mergers that may also be associated with short-GRBs or other bright optical transient sources. This will reveal not only a great deal about neutron star structure but also about the uncertain kicks in their natal supernovae. 


\section{Conclusions}

Binary population synthesis has achieved a great number of success in predicting details of observed populations but is still highly dependent on a number of uncertainties in both binary stellar evolution and the construction of synthetic stellar populations. However in light of the very final merging of two compact remnants associated with binary stars detectable by gravitational waves, we may be approaching a time when unique and strong constraints can be placed on the uncertain physics.

Acknowledgements The author would like to thank J.C.C. Wang, E.R. Stanway, L. Xiao, L.A.S. McClelland and J.C. Bray for proofreading and commenting on this chapter.

\section{Cross-References}

- Gal-Yam, A. Observation and Physical Classificiation of Supernovae

- Arcavi, I. Hydrogen-Rich Core Collapse Supernovae”

- Pian, E., Mazzali, P. Hydrogen-Poor Core Collapse Supernova

- Limongi, M., Supernovae from massive stars (12-100 Msun)

- Benvenuto, O. Bersten, M., Close Binary Stellar Evolution and Supernovae

- Van Dyk, S., Supernova Progenitors Observed with HST

- Casares, J., Israelan, G., Jonker, P., X-ray Binaries

- van der Heuvel, E., Supernovae and the Evolution of Close Binary Systems

\section{References}

1. G. Torres, J. Andersen, A. Gimnez, (2010)Accurate masses and radii of normal stars: modern results and applications. The Astronomy and Astrophysics Review, doi: 10.1007/s00159009-0025-1

2. J. Southworth (2015) DEBCat: A Catalog of Detached Eclipsing Binary Stars in Living Together: Planets, Host Stars and Binaries, ed. by S. M. Rucinski, G. Torres, M. Zeja. ASP Conference Series, Vol. 496 (San Francisco: Astronomical Society of the Pacific), p. 164.

3. O. de Marco, R.G. Izzard (2016) The impact of companions on stellar evolution. Publications of the Astronomical Society of Australia, doi: 10.1017/pasa.2016.52.

4. P.P. Eggleton (1983) Approximations to the radii of Roche lobes. Astrophysical Journal, doi: $10.1086 / 160960$

5. S.E. de Mink, N. Langer, R.G. Izzard, H. Sana, A. de Koter (2013) The Rotation Rates of Massive Stars: The Role of Binary Interaction through Tides, Mass Transfer, and Mergers. The Astrophysical Journal, doi: 10.1088/0004-637X/764/2/166

6. M. Cantiello, S.-C. Yoon, N. Langer, M. Livio (2007) Binary star progenitors of long gammaray bursts. Astronomy and Astrophysics, doi: 10.1051/0004-6361:20077115

7. N. Ivanova, S. Justham, X. Chen, O. De Marco, C.L. Fryer, E. Gaburov et al. (2013) Common envelope evolution: where we stand and how we can move forward. The Astronomy and Astrophysics Review, doi: 10.1007/s00159-013-0059-2 
8. M. Zhao, D. Gies, J.D. Monnier, N. Thureau, E. Pedretti, F. Baron et al., (2008) First Resolved Images of the Eclipsing and Interacting Binary Lyrae. The Astrophysics Journal Letters, doi: $10.1086 / 592146$

9. J. Figueiredo, J.P. De Greve, R.W. Hilditch (1994) OB-type binaries: Models versus observations Astronomy and Astrophysics, 283, 144.

10. S.E. de Mink, O.R. Pols, R.W. Hilditch (2007) Efficiency of mass transfer in massive close binaries. Tests from double-lined eclipsing binaries in the SMC. Astronomy and Astrophysics, doi: 10.1051/0004-6361:20067007

11. J.R. Hurley, C.A. Tout, O.R. Pols (2002) Evolution of binary stars and the effect of tides on binary populations. Monthly Notices of the Royal Astronomical Society, doi: 10.1046/j.13658711.2002.05038.x

12. G. Nelemans, C.A. Tout (2005) Reconstructing the evolution of white dwarf binaries: further evidence for an alternative algorithm for the outcome of the common-envelope phase in close binaries. Monthly Notices of the Royal Astronomical Society, doi: 10.1111/j.13652966.2004.08496.x

13. G. Hobbs, D.R. Lorimer, A.G. Lyne, M. Kramer (2005) A statistical study of 233 pulsar proper motions. Monthly Notices of the Royal Astronomical Society, doi: 10.1111/j.13652966.2005.09087.x

14. S. Repetto, M.B. Davies, S. Sigurdsson (2012) Investigating stellar-mass black hole kicks. Monthly Notices of the Royal Astronomical Society, doi: 10.1111/j.1365-2966.2012.21549.x

15. I. Mandel (2016) Estimates of black hole natal kick velocities from observations of lowmass X-ray binaries. Monthly Notices of the Royal Astronomical Society, doi: 10.1093/mnras/stv2733

16. N.J. Wright, J.E. Drew, M. Mohr-Smith (2015) The massive star population of Cygnus OB2. Monthly Notices of the Royal Astronomical Society, doi: 10.1093/mnras/stv323

17. M.J. Pecaut, E.E. Mamajek, E.J. Bubar. (2012) A Revised Age for Upper Scorpius and the Star Formation History among the F-type Members of the Scorpius-Centaurus OB Association. The Astrophysical Journal, doi: 10.1088/0004-637X/746/2/154

18. E.E. Salpeter (1955) The Luminosity Function and Stellar Evolution. Astrophysical Journal, doi: $10.1086 / 145971$

19. P. Kroupa, C.A. Tout, G. Gilmore (1993) The distribution of low-mass stars in the Galactic disc. Monthly Notices of the Royal Astronomical Society, doi: 10.1093/mnras/262.3.545

20. N. Bastian, K.R. Covey, M.R. Meyer (2010) A Universal Stellar Initial Mass Function? A Critical Look at Variations. Annual Review of Astronomy and Astrophysics, doi: 10.1146/annurev-astro-082708-101642

21. F.L. Villante, A.M. Serenelli, F. Delahaye, M.H. Pinsonneault (2014) The Chemical Composition of the Sun from Helioseismic and Solar Neutrino Data. The Astrophysical Journal, doi: 10.1088/0004-637X/787/1/13

22. C. Georgy, A. Granada, S. Ekström, G. Meynet, R.I. Anderson, A. Wyttenbach et al. (2014) Populations of rotating stars. III. SYCLIST, the new Geneva population synthesis code. Astronomy and Astrophysics, doi: 10.1051/0004-6361/201423881

23. G. Duchêne, A. Kraus (2013) Stellar Multiplicity. Annual Review of Astronomy and Astrophysics, doi: 10.1146/annurev-astro-081710-102602

24. A. Tokovinin, O. Kiyaeva (2016) Eccentricity distribution of wide binaries. Monthly Notices of the Royal Astronomical Society, doi: 10.1093/mnras/stv2825

25. F. Dosopoulou, V. Kalogera (2016) Orbital Evolution of Mass-transferring Eccentric Binary Systems. I. Phase-dependent Evolution. The Astrophysical Journal, doi: 10.3847/0004$637 \mathrm{X} / 825 / 1 / 70$

26. Z. Han, Ph. Podsiadlowksi, P.P. Eggleton, (1994) A Possible Criterion for Envelope Ejection in Asymptotic Giant Branch or First Giant Branch Stars. Monthly Notices of the Royal Astronomical Society, doi: 10.1093/mnras/270.1.121

27. V.M. Lipunov, K.A. Postnov, M.E. Prokhorov, A.I. Bogomazov, (2009) Description of the "Scenario Machine". Astronomy Reports, doi: 10.1134/S1063772909100047

28. D. Vanbeveren, C. De Loore, W. Van Rensbergen (1998) Massive stars. The Astronomy and Astrophysics Review, doi: 10.1007/s001590050015 
29. J.J. Eldridge, R.G. Izzard, C.A. Tout (2008) The effect of massive binaries on stellar populations and supernova progenitors. Monthly Notices of the Royal Astronomical Society, doi: 10.1111/j.1365-2966.2007.12738.x

30. P. Marchant, N. Langer, P. Podsiadlowski, T.M. Tauris, T.J. Moriya (2016) A new route towards merging massive black holes. Astronomy and Astrophysics, doi: 10.1051/0004$6361 / 201628133$

31. J.C. Bray, J.J. Eldridge (2016) Neutron star kicks and their relationship to supernovae ejecta mass. Monthly Notices of the Royal Astronomical Society, doi: 10.1093/mnras/stw1275

32. B. Müller (2016) Publications of the Astronomical Society of Australia, in press.

33. B.M. Tinsley (1975) What stars become supernovae. Astronomical Society of the Pacific, Publications, doi: 10.1086/129857

34. S.J. Smartt (2015) Observational Constraints on the Progenitors of Core-Collapse Supernovae: The Case for Missing High-Mass Stars. Publications of the Astronomical Society of Australia, doi: 10.1017/pasa.2015.17

35. J. Whelan, I. Iben, Jr. (1973) Binaries and Supernovae of Type I. Astrophysical Journal, doi: $10.1086 / 152565$

36. I. Iben, Jr., A. V. Tutukov (1984) Supernovae of type I as end products of the evolution of binaries with components of moderate initial mass (M not greater than about 9 solar masses). Astrophysical Journal Supplement Series, doi: 10.1086/190932

37. R. F. Webbink (1984) Double white dwarfs as progenitors of $R$ Coronae Borealis stars and Type I supernovae. Astrophysical Journal, doi: 10.1086/161701

38. C. McCully, S.W. Jha, R.J. Foley, L. Bildsten, W.-F. Fong, R.P. Kirshner et al., (2014) A luminous, blue progenitor system for the type Iax supernova 2012Z. Nature, doi: 10.1038/nature 13615

39. Ph. Podsiadlowski, P.C. Joss, J.J.L. Hsu (1992) Presupernova evolution in massive interacting binaries. Astrophysical Journal, doi: 10.1086/171341

40. E. De Donder, D. Vanbeveren (1998) The relative frequency of type II and $\left.I_{(} b, c\right)$ supernovae and the birth rate of double compact star binaries. Astronomy and Astrophysics 333, 557

41. R.G. Izzard, E. Ramirez-Ruiz, C.A. Tout (2004) Formation rates of core-collapse supernovae and gamma-ray bursts. Monthly Notices of the Royal Astronomical Society, doi: 10.1111/j.1365-2966.2004.07436.x

42. S.-C. Yoon, S.E. Woosley, N. Langer (2010) Type Ib/c Supernovae in Binary Systems. I. Evolution and Properties of the Progenitor Stars. The Astrophysical Journal, doi: 10.1088/0004$637 X / 725 / 1 / 940$

43. J.J. Eldridge, M. Fraser, S.J. Smartt, J.R. Maund, R.M. Crockett (2013) The death of massive stars - II. Observational constraints on the progenitors of Type Ibc supernovae. Monthly Notices of the Royal Astronomical Society, doi: 10.1093/mnras/stt1612

44. S.-C. Yoon, G. Grfener, J.S. Vink, A. Kozyreva, A., R.G. Izzard (2012) On the nature and detectability of Type Ib/c supernova progenitors. Astronomy and Astrophysics, doi: 10.1051/0004-6361/201219790

45. Y. Cao, M.M. Kasliwal, I. Arcavi, A. Horesh, P. Hancock, S. Valenti et al. (2013) Discovery, Progenitor and Early Evolution of a Stripped Envelope Supernova iPTF13bvn. The Astrophysical Journal Letters, doi: 10.1088/2041-8205/775/1/L7

46. M. Bertsen, O.G. Benvenuto, G. Folatelli, K. Nomoto, H. Kuncarayakti, S. Srivastav et al. (2014) iPTF13bvn: The First Evidence of a Binary Progenitor for a Type Ib Supernova. The Astronomical Journal, doi: 10.1088/0004-6256/148/4/68

47. J.H. Groh, C. Georgy, S. Ekström (2013) Progenitors of supernova Ibc: a single Wolf-Rayet star as the possible progenitor of the SN Ib iPTF13bvn. Astronomy and Astrophysics, doi: 10.1051/0004-6361/201322369

48. J.J. Eldridge, J.R. Maund (2016) The disappearance of the helium-giant progenitor of the Type Ib supernova iPTF13bvn and constraints on its companion. Monthly Notices of the Royal Astronomical Society: Letters, doi: 10.1093/mnrasl/slw099

49. Zapartas, E.; de Mink, S. E.; Izzard, R. G.; Yoon, S.-C.; Badenes, C.; Gotberg, Y.; (2017) Delay-time distribution of core-collapse supernovae with late events resulting from binary interaction. Astronomy \& Astrophysics in press. 
50. K. Belczynski, G. Wiktorowicz, C.L. Fryer, D.E. Holz, V. Kalogera (2012) Missing Black Holes Unveil the Supernova Explosion Mechanism, The Astrophysical Journal, doi: 10.1088/0004-637X/757/1/91

51. R.P. Church, C. Kim, A.J. Levan, M.B. Davies (2012) The properties of long gamma-ray bursts in massive compact binaries. Monthly Notices of the Royal Astronomical Society, doi: 10.1111/j.1365-2966.2012.21480.x

52. P.C. Peters (1964) Gravitational Radiation and the Motion of Two Point Masses. Physical Review, doi: 10.1103/PhysRev.136.B1224

53. LIGO Collaboration (2016) Observation of Gravitational Waves from a Binary Black Hole Merger. Physics Review Letters, doi: 10.1103/PhysRevLett.116.061102

54. K. Belczynski, M. Dominik, T. Bulik, R. O'Shaughnessy, C. Fryer, D.E. Holz (2010) The Effect of Metallicity on the Detection Prospects for Gravitational Waves. The Astrophysical Journal Letters, doi: 10.1088/2041-8205/715/2/L138

55. S.E. de Mink, K. Belczynski (2015) Merger Rates of Double Neutron Stars and Stellar Origin Black Holes: The Impact of Initial Conditions on Binary Evolution Predictions. The Astrophysical Journal, doi: 10.1088/0004-637X/814/1/58

56. V.M. Lipunov, M.V. Pruzhinskaya (2014) Scenario Machine: fast radio bursts, short gammaray burst, dark energy and Laser Interferometer Gravitational-wave Observatory silence. Monthly Notices of the Royal Astronomical Society, doi: 10.1093/mnras/stu313

57. N. Mennekens, D. Vanbeveren (2016) The delay time distribution of massive double compact star mergers. Astronomy and Astrophysics, doi: 10.1051/0004-6361/201628193

58. K. Belczynski, D.E. Holz, T. Bulik, R. OShaughnessy (2016) The first gravitational-wave source from the isolated evolution of two stars in the 40100 solar mass range. Nature, doi: 10.1038 /nature 18322

59. I. Mandel, S.E. de Mink (2016) Merging binary black holes formed through chemically homogeneous evolution in short-period stellar binaries. Monthly Notices of the Royal Astronomical Society, doi: 10.1093/mnras/stw379

60. J.J Eldridge, E.R. Stanway (2016) BPASS predictions for binary black hole mergers. Monthly Notices of the Royal Astronomical Society, doi: 10.1093/mnras/stw1772 